MATHEMATICAL LOGIC IN THE 20TH CENTURY

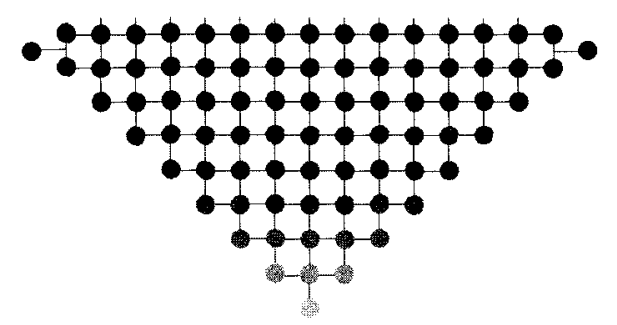


This page is intentionally left blank 


\section{MATHEMATICAL LOGIC IN THE 20TH CENTURY}

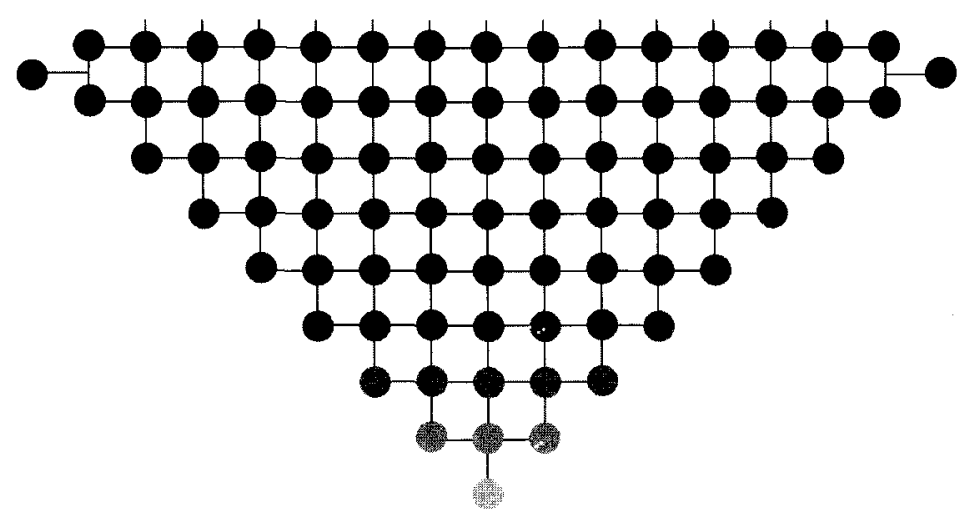

Gerald E. Sacks 


\section{Published by}

Singapore University Press

Yusof Ishak House, National University of Singapore

31 Lower Kent Ridge Road, Singapore 119078

and

World Scientific Publishing Co. Pte. Ltd.

5 Toh Tuck Link, Singapore 596224

USA office: Suite 202, 1060 Main Street, River Edge, NJ 07661

UK office: 57 Shelton Street, Covent Garden, London WC2H 9HE

\section{British Library Cataloguing-in-Publication Data}

A catalogue record for this book is available from the British Library.

\section{MATHEMATICAL LOGIC IN THE 20TH CENTURY}

Copyright (C) 2003 by Singapore University Press and World Scientific Publishing Co. Pte. Ltd.

All rights reserved. This book, or parts thereof, may not be reproduced in any form or by any means, electronic or mechanical, including photocopying, recording or any information storage and retrieval system now known or to be invented, without written permission from the Publishers.

For photocopying of material in this volume, please pay a copying fee through the Copyright Clearance Center, Inc., 222 Rosewood Drive, Danvers, MA 01923, USA. In this case permission to photocopy is not required from the publishers.

ISBN 981-02-4736-2

Printed in Singapore. 
For Jacob, Ella and Maggie 
This page is intentionally left blank 


\section{Introduction}

Brevity is the soul.

H. M.

First come the disclaimers, then the rules for selecting the papers, the exceptions to the rules, the justifications of the exceptions, and finally some brief remarks on the papers. (But before all that, the point of it all. The original papers are studied in the hope of recovering early ideas lost in later expositions. Proofs are rare, but the ideas used in proofs are rarer still.) The title of this volume is too broad. Almost all of the papers belong to the second half of the twentieth century. The last decade of the twentieth century is lightly represented. Only so much can be forced into one volume. The first half of the last century is well represented elsewhere; now is too soon to reach conclusions about its final decade.

I have not read all the logic of the last century - far from it. And only a fraction of what was read was understood. The choices made were personal in nature. Who knows what "personal" means? The selection was certainly not based on an Olympian view of mathematical logic derived from a long and scholarly life of pondering the subject. Perhaps the choices cohere, if only because it is hard to see how it could be otherwise.

The selection rules were:

R1. No papers from before World War II.

R2. No long papers.

R3. At most one paper by any author.

R4. The paper was (and is) intellectually exciting then (and now).

The first three rules are justified above. They proved difficult to follow. Gödel's pre-war National Academy paper on the generalized continuum hypothesis is short, readable, more illuminating than his subsequent Princeton Mathematics orange book, and probably closer to his early thinking. His Academy paper, but not his orange book, mentions Russell's Axiom of Reducibility as a source of inspiration. The papers by Kleene and Tarski are much too long for this volumn, but Kleene is the father of recursion theory and Tarski of model theory. Why this particular paper by Kleene? Two reasons. He lifts the concepts of classical recursion theory to objects of finite type, and he shows that sets of non-negative integers are hyperarithmetic if and only if they are recursive in ${ }^{2} E$, the type 2 object corresponding to the number quantifier. The title of Tarski's paper speaks for itself. Rule R4 was not violated, possibly a necessary fact.

Cohen has two papers intended for this volume, but they are in fact the two halves of one paper. For this work, he received the Fields Medal, the highest international award in mathematics.

Gödel's paper transformed set theory into a subject that welcomes a wide range of mathematical ideas. His use of the downward Skolem Löwenheim theorem inside 
$\mathrm{L}$ is the beginning of fine structure theory. His paper combined with Cohen's puts Cantor's continuum problem outside the conventional realms (ZFC) of set theory.

Cohen's paper introduced the method of forcing, an essential technique with applications throughout logic. Forcing has unconscious precursors in recursion theory, for example the construction of a minimal Turing degree in Spector's paper.

Silver's paper proved (in ZFC) a new theorem of cardinal arithmetic at a time when such an outcome was thought unlikely because of the Gödel and Cohen results. He applied some ideas about ultrafilters to show: if the generalized continuum hypothesis holds below a singular cardinal $\kappa$ of uncountable cofinality, then it holds at $\kappa$. This line of thought led to Shelah's pcf theory [11], which yields estimates on the size of the power set of a singular cardinal of countable confinality. ([Im] is the $m$ th item in the References at the end of this introduction. All other papers mentioned are from the Contents list for the volume.)

Choosing a paper by Shelah was a daunting task because of the large number of his contributions to model theory and set theory and the limit imposed by rule R2. His 1969 paper on stability can be seen as the beginning of his sweeping transformation of model theory.

The notion of $\omega$-stability originated in Morley's proof that a countable theory categorical in some uncountable power is categorical in all uncountable powers. Morley's paper, building on Vaught's earlier paper, was the beginning of modern model theory. Vaught's paper went beyond immediate applications of compactness and stressed the notion of element type. For example he showed that the number of countable models up to isomorphism of a complete countable theory could not be two.

Jensen's covering theorem, in his paper with Devlin, makes a connection between sets of ordinals in $V$ and $L$ with the help of fine structure theory and the work of Silver [I2] and Solovay [I3] on $O^{\sharp}$.

The choice of Friedberg's paper on recursive enumeration came about as follows. $\mathrm{He}$ in [I4] and Mučnik independently solved Post's problem by introducing the priority method, a technique that dominates classical recursion theory to this day. By choosing Mučnik's version, I could satisfy rule R3 and still include Friedberg's construction of a maximal recursively enumerable set, a result that ignited interest in the lattice of recursively enumerable set under inclusion modulo finite sets. The lattice was initially studied in Post's paper on recursively enumerable sets and their decision problems. The lasting influence of Post's paper entitles him to be called the co-father, if there is such a thing, with Kleene of recursion theory. Soare's paper showed any two maximal sets are automorphic. His result is the reason that the lattice continues to be of interest.

Post's paper established the legitimacy of an intuitive approach to recursion theory: less equations and more words. Friedberg's paper is any early example of the intuitive style. Spector's paper adheres to Kleene's formal style, only because it was extracted from Spector's thesis supervised by Kleene.

Lachlan's paper introduced the so-called (but not by him) monstrous injury method, close to the final stage in the development of the Friedberg-Mučnik priority method.

Moschovakis's paper found a nearly paradoxical role for divergence in recursion theory and led to constructions of recursively enumerable sets in higher recursion theory in which divergence witnesses played as big a part as convergence witnesses. 
Matijasevič's paper on the unsolvability of the Diophantine problem has historical antecedents as old as any in mathematics.

The underrepresentation of proof theory in this volume indicates nothing more than my own confusion over the subject. The most striking proof theorist I have met is Girard. His paper was chosen for its brevity and as an example of his unique mode of thought. In it he discusses his concept of dilator.

Kreisel, another proof theorist with his own mode of thought, is also included. His paper is a mixture of recursion theory, model theory, proof theory and other subjects hard to put a name to. He presents a compactness theorem for $\omega$-logic based on his insight that generalizing the notion of finite is the key to extending various results in model theory and recursion theory. In this compactness theorem "hyperarithmetic" is the generalization of "finite".

Robinson's paper on non-standard analysis is a model theorist's way of making sense out of infinitesimals. Gödel thought it was of historic importance.

Wilkie's paper solves a long standing problem of Tarksi on the first order theory of the reals with the exponential function added.

The work of Zil'ber and Hruschovski bring ideas of geometry and stability to bear on model theory. Zil'ber's paper was chosen as a brief example of his approach, and Hruschovski's paper as a prime application of model theory to number theory.

H. Friedman's paper shows that Borel Determinateness (BD) cannot be proved without invoking objects of arbitrarily high countable rank despite the fact that $\mathrm{BD}$ is about Borel sets of reals, objects of rank 1. Later Martin [I5] proved BD by means of an induction that trades decreases in rank of Borel sets for increases in rank of objects.

Solovay's paper assumes the consistency of "there exists an inaccessible cardinal" and then demonstrates the consistency of "every set of reals is Lebesgue measurable and countable dependent choice". Later Shelah [I6] proved the converse.

Scott's paper showed the existence of a measurable cardinal implies the existence of a non-constructible set. This result, and its proof via ultrapowers, broke open the study of large cardinals.

Martin's paper used a measurable cardinal to establish the determinacy of analytic games. His argument needed only the sort of indiscernibles provided by $O^{\sharp}$. Later the converse was shown by Harrington [I7]. (Thus $O^{\natural}$ is equivalent to lightface $\Pi_{1}^{1}$ determinacy.) Martin's result eventually led to complex connections between determinateness and large cardinals obtained by Woodin, whose paper in this volume is a brief example of his unique insight.

Shoenfield's $\Sigma_{2}^{1}$ absoluteness result is a personal favorite. It has applications throughout logic. One example is the Slaman-Woodin proof [I8] of the definability of the double Turing jump.

My thanks to those who suggested papers for this volume. They insist on remaining anonymous. 


\section{References for the Introduction}

[I1] S. Shelah, Cardinal Arithmetic, Oxford Logic Guides 29, Oxford Science Publications, The Clarendon Press, Oxford University Press, New York 1994.

[I2] J. Silver, Some applications of model theory in set theory, Ann. Math. Logic 3 (1971) no. 1, 45-110.

[I3] R. Solovay, A nonconstructible $\Delta_{3}^{1}$ set of integers, Trans. Amer. Math. Soc. 127 (1967), 50-75.

[I4] R. Friedberg, Two recursively enumerable sets of incomparable degrees of unsolvability, Proc. Nat. Acad. Sci. USA 43 (1957), 236-238.

[I5] D. A. Martin, Borel determinacy, Ann. of Math., Ser. 2, 102 (1975) no. 2, $363-371$.

[I6] S. Shelah, Can you take Solovay's inaccessible away?, Israel J. Math. 48 (1984), no. 1, 1-47.

[I7] L. Harrington, Analytic determinacy and $O^{\sharp}$, J. Symbolic Logic 43 (1987), no. $4,685-693$.

[I8] T. Slaman and H. Woodin, Definability in degree structures, forthcoming. 


\section{Contents}

Introduction

The Independence of the Continuum Hypothesis

Cohen, Paul J.

The Independence of the Continuum Hypothesis II Cohen, Paul J.

Marginalia to a Theorem of Silver Devlin, K. I. and Jensen, R. B.

Three Theorems on Recursive Enumeration. I. Decomposition.

II. Maximal Set. III. Enumeration without Duplication Friedberg, Richard $M$.

Higher Set Theory and Mathematical Practice Friedman, Harvey $M$.

Introduction to $\Pi_{2}^{1}$-Logic

Girard, Jean-Yves

Consistency-Proof for the Generalized Continuum-Hypothesis Gödel, Kurt

The Mordell-Lang Conjecture for Function Fields Hrushovski, Ehud

Model-Theoretic Invariants: Applications to Recursive and

Hyperarithmetic Operations

Kreisel, G.

Recursive Functionals and Quantifiers of Finite Types I Kleene, S. C.

A Recursively Enumerable Degree which will not Split over all Lesser Ones

Lachlan, A. H.

Measurable Cardinals and Analytic Games

Martin, Donald $A$. 
$\begin{array}{ll}\text { Enumerable Sets are Diophantine } & 269\end{array}$

Matijasevič, Ju. V.

Categoricity in Power

Morley, Michael

Hyperanalytic Predicates

Moschovakis, $Y . N$.

Solution of Post's Reduction Problem and Some Other Problems

of the Theory of Algorithms

Mučnik, A. A.

Recursively Enumerable Sets of Positive Integers and Their

Decision Problems Post, Emil L.

Non-Standard Analysis

Robinson, Abraham

The Recursively Enumerable Degrees are Dense Sacks, Gerald E.

Measurable Cardinals and Constructible Sets

Scott, Dana

Stable Theories

Shelah, $S$.

The Problem of Predicativity

Shoenfield, J. R.

On the Singular Cardinals Problem

Silver, Jack

Automorphisms of the Lattice of Recursively Enumerable Sets

Part I: Maximal Sets

Soare, Robert

A Model of Set-Theory in which Every Set of Reals is Lebesgue

Measurable

Solovay, Robert $M$.

On Degrees of Recursive Unsolvability Spector, Clifford

A Decision Method for Elementary Algebra and Geometry 
Denumerable Models of Complete Theories

Vaught, R. L.

Model Completeness Results for Expansions of the Ordered Field of Real Numbers by Restricted Pfaffian Functions and the Exponential Function

Wilkie, A. J.

Supercompact Cardinals, Sets of Reals, and Weakly

Homogeneous Trees

Woodin, W. Hugh

Structural Properties of Models of $\aleph_{1}$-Categorical Theories Zil'ber, B. I.

Permissions 